\title{
Automatic Category Structure Generation and Categorization of Chinese Text Documents
}

\author{
Hsin-Chang Yang and Chung-Hong Lee \\ Department of Information Management, Chang Jung University, \\ Tainan, 711, Taiwan \\ \{hcyang, leechung\}@mail.cju.edu.tw
}

\begin{abstract}
Recently knowledge discovery and data mining in unstructured or semi-structured texts(text mining) has been attracted lots of attention from both commercial and research fields. One aspect of text mining is on automatic text categorization, which assigns a text document to some predefined category according to the correlation between the document and the category. Traditionally the categories are arranged in hierarchical manner to achieve effective searching and indexing as well as easy comprehension for human. The determination of categories and their hierarchical structures were most done by human experts. In this work, we developed an approach to automatically generate categories and reveal the hierarchical structure among them. We also used the generated structure to categorize text documents. The document collection is trained by a self-organizing map to form two feature maps. We then analyzed the two maps to obtain the categories and the structure among them. Although the corpus contains documents written in Chinese, the proposed approach can be applied to documents written in any language and such documents can be transformed into a list of separated terms.
\end{abstract}

\section{Introduction}

Text categorization concerns about assigning a text document to some predefined category. When a set of documents are well categorized, both storage and retrieval of these documents can be effectively achieved. A primary characteristic of text categorization is that a category reveal the theme of those documents under this category, that is, these documents form a natural cluster of similar context. Thus text categorization provides some knowledge about the document collection. An interesting argument about text categorization is that before we can acquiring knowledge through text categorization, we need some knowledge to correctly categorize documents. One of the key knowledge we need to perform text categorization is the generation of categories and the structure among categories. Traditionally such knowledge is provided by human experts or some semi-automatic mechanisms which incorporate human knowledge as well as computing techniques, for example, natural language processing. Fully automatic category generation is difficult due to two reasons. First, we need to select some important words that can be assigned as category terms(or labels). We

D.A. Zighed, J. Komorowski, and J. Żytkow (Eds.): PKDD 2000, LNAI 1910, pp. 673-678, 2000. (C) Springer-Verlag Berlin Heidelberg 2000 
use these words to represent categories and provide indexing information for the categorized documents. A proper selection of a term should represent the general theme of the documents under the corresponding category. Such selections were always done by human linguistic experts because we need an insight of the underlying semantic structure of a language. Such insight is hard to automate. Certain techniques such as word frequency counts may help, but it is the human experts to decide which terms are most discriminative and representative. Second, the categories were always arranged in a tree-like hierarchical structure. This hierarchy reveals the relationships among categories. A category associated to higher level nodes of the hierarchy represents a more general theme than those associated to lower level nodes. The parent category in the tree should represent the common theme of its child categories. Thus the retrieval of documents of a particular interest can be effectively achieved through such hierarchy. The hierarchy must be constructed carefully so that irrelevant categories may not be the children of the same parent category. A thorough investigation about the semantic relations among category terms must be conducted to establish a well-organized hierarchy. Therefore, most of text categorization systems focus on categorizing documents according to some human-specified category terms and hierarchy, rather than on generating category terms and hierarchy.

In this work, we provide a method which can automatically generate category terms and establish category structure. Traditionally, category terms are selected according to the popularity of words in the majority of documents. This can be done by human engineering, statistical training, or a combination of the two. In this work, we reverse the text categorization process to obtain the category terms. First we should cluster the documents. The document collection is trained by the self-organizing maps (SOM) 1] algorithm to generate two feature maps, namely the document cluster map and the word cluster map. A neuron in these two maps represents a document cluster and a word cluster respectively. Through self-organizing process the distribution of neurons in the map reveals the similarities among clusters. We select category terms according to such similarities. To generate the category terms, dominating neurons in the document cluster map are first selected as centroids of some super-clusters, which each represents a general category. The words associated to the corresponding neurons in word cluster map are then used to select category terms. The structure of categories may also be revealed by examining the correlations among neurons in the two maps.

The corpus used to train the maps consists of documents written in Chinese. We decide to use Chinese corpus by two reasons. First, over a quarter of population on earth use Chinese as their native language. However, techniques for mining Chinese documents are relatively less than for documents written in English. Second, traditional text mining (and other related fields) techniques developed based on English corpora are not quite suitable for processing Chinese documents. Nowadays, demands for Chinese-based text mining techniques arise rapidly by many commercial fields. A difficult problem in developing Chinesebased text mining techniques is that research on lexical analysis of Chinese 
documents is still in its infancy. Besides, there is still no attempt in discovering knowledge from such a large repository so far. Therefore methodologies developed for English documents play an inevitable role in developing a model for knowledge discovery in Chinese documents. In this work, traditional term-based representation scheme in information retrieval field is adopted for document encoding. The same method developed in our work can naturally extend to English documents because these documents can always be represented by a list of terms.

\section{Related Works}

Text categorization or classification systems usually categorize documents according to some predefined category hierarchy. An example is the works by CMU text learning group [2] where they used Yahoo hierarchy to categorize documents. Another approach is to automatically generate the category terms as well as hierarchy. McCallum and Nigam [3] used a bootstrapping process to generate new terms from a set of human-provided keywords. Human intervention is still required in their work. Rauber and Merkl [4] used the self-organizing map to cluster text documents. They labeled each neuron with a set of keywords that were selected from the input vectors mapped to this neuron. Those keywords that contribute less quantization error were selected. These keywords together form the label of a cluster, rather than a single term for a category. Moreover, the hierarchical structure among these keywords were not revealed.

\section{Automatic Category Hierarchy Generation}

To obtain the category hierarchy, we first cluster documents by SOM using the same data set and method in [5] to generate two cluster maps, namely the document cluster map and the word cluster map. A neuron in the document cluster map represents a cluster of documents. Documents associated with neighboring neurons contain words that often co-occur in these documents. Thus we may form a super-cluster by combining neighboring neurons. To form a super-cluster, we first define distance between two clusters: $D(i, j)=\left\|\mathbf{G}_{i}-\mathbf{G}_{j}\right\|$, where $i$ and $j$ are the neuron indices of the two clusters and $\mathbf{G}_{i}$ is the two-dimensional grid location of neuron $i .\left\|\mathbf{G}_{i}-\mathbf{G}_{j}\right\|$ measures the Euclidean distance between the two coordinates $\mathbf{G}_{i}$ and $\mathbf{G}_{j}$. We also define the dissimilarity between two clusters: $\mathcal{D}(i, j)=\left\|\mathbf{w}_{i}-\mathbf{w}_{j}\right\|$, where $\mathbf{w}_{i}$ is the synaptic weight vector of neuron $i$. We may compute the supporting cluster similarity $\mathcal{S}_{i}$ for a neuron $i$ from its neighboring neurons by

$$
\begin{aligned}
S(i, j) & =\frac{\operatorname{doc}(i) \operatorname{doc}(j)}{F(D(i, j) \mathcal{D}(i, j))} \\
\mathcal{S}_{i} & =\sum_{j \in B_{i}} S(i, j),
\end{aligned}
$$

where $\operatorname{doc}(i)$ is the number of documents associated to neuron $i$ in the document cluster map and $B_{i}$ is the set of neuron index in the neighborhood of neuron 
$i$. The function $F: \mathbf{R}^{+} \rightarrow \mathbf{R}^{+}$is a monotonically increasing function. A dominating neuron is the neuron which has locally maximal supporting cluster similarity. We may select dominating neuron by the following algorithm:

1. Find the neuron with the largest supporting cluster similarity. Selecting this neuron as dominating neuron.

2. Eliminate its neighbor neurons so that they will not be considered as dominating neurons.

3. If there is no neuron left or the number of dominating neurons exceeds a predetermined value, stop. Otherwise goto step 1.

A dominating neuron is the centroid of a super-cluster, which contains several clusters. The $i$ th cluster (neuron) belongs to the $k$ th super-cluster if $\mathcal{D}(i, k)=$ $\min _{l} \mathcal{D}(i, l)$, where $l$ is a super-cluster. A super-cluster may be thought as a category which contains several sub-categories. Let $C_{k}$ denote the set of neurons that belong to the $k$ th super-cluster. The category terms are selected from words in the word cluster map that associated to neurons in $C_{k}$. For all neurons $j \in C_{k}$, we select the $n^{*}$ th word as the category term if

$$
\sum_{j \in C_{k}} w_{j_{n^{*}}}=\max _{1 \leq n \leq N} \sum_{j \in C_{k}} w_{j_{n}} .
$$

Eq. 2 selects the term that is the most important to a super-cluster since the weight of a synapse in a neuron reflects the willingness that the neuron wants to learn the corresponding input data, i.e. a word in our case.

The terms selected by Eq. 2 form the root nodes of the category hierarchies. The number of generated hierarchies is the same as the number of super-clusters. To find the children of these root nodes, we may apply the above process to each super-cluster. A set of sub-category will be obtained for each hierarchy. These sub-categories form the new super-clusters that are the children of the root node of the hierarchy. The category structure can then be revealed by recursively applying the same category generation process to each new-found super-cluster. We decrease the size of neighborhood in selecting dominating neurons when we try to find the sub-categories. In each category hierarchy, each leaf node represents an individual neuron in the trained map.

\section{Automatic Text Categorization}

A text document can be categorized into the developed category hierarchy as follows. An incoming document $T$ with document vector $\mathbf{x}_{T}$ is compared to all neurons in the trained map to find its document cluster. The neuron with synaptic weight vector which is the closest to $\mathbf{x}_{T}$ is selected. The incoming document is categorized into the category where the neuron been associated to as a leaf node in one of the category hierarchies. Through this way, we can see that the task of text categorization has been done naturally by the category generation process. 


\section{$5 \quad$ Experimental Results}

We applied our method on the Chinese news articles posted daily in the web by CNA(Central News Agency). In the preliminary experiments a corpus was constructed by randomly selecting 100 news articles posted in Aug. 1, 2, and 3, 1996. A word extraction process was applied to the corpus to extract Chinese words. Total 1475 words were extracted. To reduce the dimensionality of the feature vectors we discarded those words which occur only once in a document. This reduced the number of words to 563 . We also constructed a self-organizing map which contains 64 neurons in $8 \times 8$ grid format. The number of neurons was determined experimentally such that a better clustering can be achieved. Each neuron contains 563 synapses. The initial training gain was set to 0.4 and the maximal training time was set to 100 . After training we labeled the map by documents and words respectively, and obtained the document cluster map and the word cluster map. We applied the category generation process to the document cluster map to obtain the category hierarchies. Fig. 11 depicts the result after the first application of the generation process. Each circle represents a neuron in the maps. The neuron indices are shown at the upper-left corner of each neuron. The size of a circle depicts the supporting similarity of the corresponding neuron obtained by Eq. 1. A dominating neuron for a supercluster is marked by a cross. The 2-tuple $(x, y)$ beneath each circle shows that the corresponding neuron(cluster) belongs to the super-cluster $x$ where $y$ is its supporting similarity. For example, the 35th neuron has a 2 -tuple $(1,3.0)$ and a cross on it, which shows that this neuron is a dominating neuron for super-cluster 1. The supporting similarity for this neuron is 3.0. Neurons belonging to the same super-cluster locate closely in Fig. 1. The category terms for super-clusters are shown beneath the 2-tuple. Fig. 2 depicts the final category hierarchy. Each tree depicts a category hierarchy where the number in the root node depicts the super-cluster found in Fig. 1. Each node in a tree represents a cluster in the document cluster map. The parent node of some child nodes represents a super-cluster. The number enclosed in every leaf node is the neuron index of its associated cluster in the document cluster map. The root node of each tree is the super-cluster found in the first application of category generation process.

\section{Conclusions}

In this paper, we present a method to automatically generate category terms and hierarchies. The documents were first transformed to a set of feature vectors. The vectors were used as input to train the self-organizing map. Two maps, namely the word cluster map and the document cluster map, were obtained by labeling the neurons in the map with words and documents respectively. An automatic category generation process was applied to the document cluster map to find some dominating neurons that are centroids of some super-clusters. The category terms of super-clusters were also determined. The same process were applied recursively to each super-clusters to reveal the structure of the categories. Our 
method used neither human-provided terms nor predefined category structure. Text categorization can easily be achieved in our method.

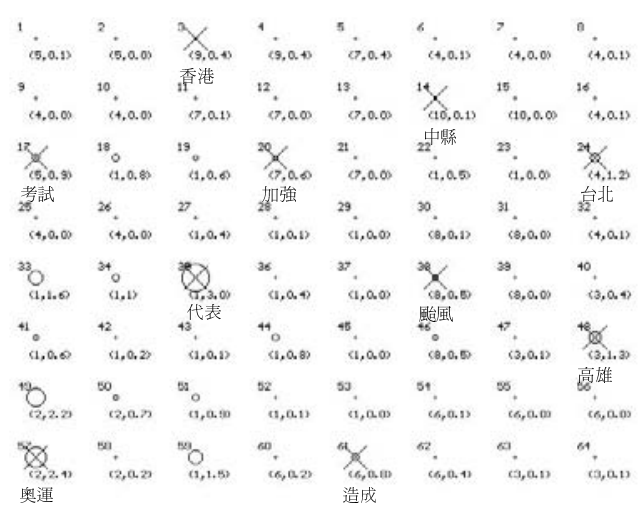

Fig. 1. The supporting similarity for each neuron in the first application of category generation process.

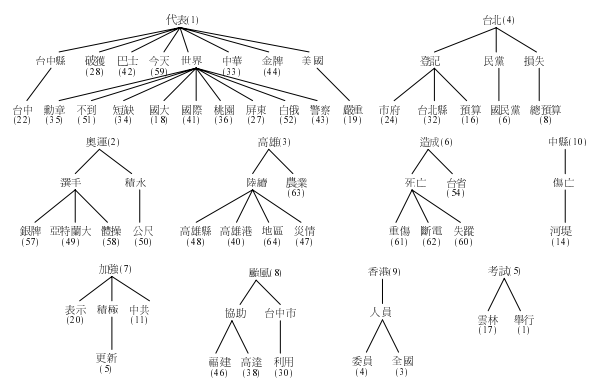

Fig. 2. The category hierarchies of the training documents.

\section{References}

1. Kohonen, T.:Self-Organizing Maps. Springer-Verlag, Berlin Heidelberg New York (1997)

2. Grobelnik, M., Mladenić, D.:Efficient Text Categorization. Proceedings of Text Mining Workshop on ECML-98, Chemnitz, Germany (1998)

3. McCallum, A., Nigam, K.:Text Classification by Bootstrapping with Keywords, EM and Shrinkage. Proceedings of ACL '99 Workshop for Unsupervised Learning in Natural Language Processing (1999) 52-58

4. Rauber, A., Merkl, D.:Using Self-Organizing Maps to Organize Document Archives and to Characterize Subject Matter: How to Make a Map Tell the News of the World. Proceedings of 10th International Conference on Database and Expert Systems Applications. Florence, Italy (1999) 302-311

5. Lee, C. H., Yang, H. C.:A Web Text Mining Approach Based on Self-Organizing Map. Proceedings of ACM CIKM'99 2nd Workshop on Web Information and Data Management. Kansas City, Missouri, USA (1999) 59-62 